\title{
Symmetries, horizons, and black hole entropy
}

\section{S. Carlip}

\begin{abstract}
Black holes behave as thermodynamic systems, and a central task of any quantum theory of gravity is to explain these thermal properties. A statistical mechanical description of black hole entropy once seemed remote, but today we suffer an embarrassment of riches: despite counting very different states, many inequivalent approaches to quantum gravity obtain identical results. Such "universality" may reflect an underlying two-dimensional conformal symmetry near the horizon, which can be powerful enough to control the thermal characteristics independent of other details of the theory. This picture suggests an elegant description of the relevant degrees of freedom as Goldstone-boson-like excitations arising from symmetry breaking by the conformal anomaly.
\end{abstract}

\section{The problem of universality}

Black holes are thermal systems, radiating as black bodies with characteristic temperatures and entropies. Classically, this behavior is a mystery: by Wheeler's famous dictum, "black holes have no hair", no classical degrees of freedom to account for such thermodynamic properties. The likely explanation is that the relevant microscopic degrees of freedom are fundamentally quantum mechanical. Indeed, the BekensteinHawking entropy

$$
S=\frac{A_{\text {horizon }}}{4 \hbar G}
$$

First Award in the 2007 Essay Competition of the Gravity Research Foundation.

S. Carlip ( $\varangle)$

Department of Physics, University of California, Davis, CA 95616, USA

e-mail: carlip@physics.ucdavis.edu 
depends upon both Planck's constant $\hbar$ and Newton's constant $G$, hinting that black hole thermodynamics unites quantum mechanics and gravity.

Until recently, little was known about such quantum degrees of freedom. Today, we suffer an embarrassment of riches. Black hole thermodynamics can be explained by

- weakly coupled string and D-brane states [1];

- states of a dual conformal field theory "at infinity" [2];

- horizonless "fuzzball" geometries [3];

- $\quad$ spin network states at [4] or inside [5] the horizon;

- "heavy" degrees of freedom in induced gravity [6];

- elements of a causal set in the horizon's domain of dependence [7];

- inherently global characteristics [8];

- entanglement entropy of quantum fields across the horizon $[9,10]$.

None of these accounts is complete; string theory, for instance, is most reliable for supersymmetric black holes, while the loop quantum gravity calculations depend on the poorly understood Immirzi parameter. But within their domains of applicability, all seem to work.

This "problem of universality" is already present within particular models. The simplest string theory approach, for example, counts weakly coupled brane configurations. But this computation does not yield the Bekenstein-Hawking formula (1) directly; rather, one must separately determine the entropy and the horizon area in terms of a set of charges, and then check, case by case, that they match. Similarly, loop quantum gravity uses special features of four-dimensional spacetimes; it works for many different $(3+1)$-dimensional black holes, but does not explain what happens in other dimensions. More generally, in the absence of classical degrees of freedom to which one could apply the correspondence principle, it is not clear why any counting of microstates should reproduce Hawking's original results [11], which were based on quantum field theory in a fixed, classical gravitational background.

\section{Symmetries and microstates}

We do not know why such disparate computations yield the same black hole entropy. But one intriguing possibility is that a classical symmetry near the horizon may govern the number of degrees of freedom, independent of the details of quantum gravity.

To an observer outside a black hole, the near-horizon region is effectively twodimensional and conformally invariant $[12,13]$ : transverse excitations and dimensionful quantities are red-shifted away relative to the degrees of freedom in the $r-t$ plane. Thermodynamic quantities such as temperature are conformally invariant [14]; indeed, a conformal description is powerful enough to determine the flux [15] and spectrum [16] of Hawking radiation.

Remarkably, such a symmetry may also provide a universal explanation of black hole entropy. As Cardy has shown [17,18], the entropy of a two-dimensional conformal field theory is completely fixed by a few parameters determined by the symmetry. More precisely, the fundamental symmetry of a two-dimensional conformal field theory is invariance under holomorphic coordinate transformations $z \rightarrow z+\xi(z)$, 
$\bar{z} \rightarrow \bar{z}+\bar{\xi}(\bar{z})$. The Poisson brackets of the generators of such transformations form a Virasoro algebra

$$
\{L[\xi], L[\eta]\}=L[\xi \dot{\eta}-\eta \dot{\xi}]+\frac{c}{48 \pi} \int \mathrm{d} t(\dot{\xi} \ddot{\eta}-\dot{\eta} \ddot{\xi})
$$

with a corresponding expression for $\bar{L}[\bar{\xi}]$. The first term on the right-hand side gives the ordinary commutator of vector fields. The second is the unique central extension, completely characterized by the "central charge" $c$. Like other symmetries, conformal invariance implies the existence of conserved charges: the zero-mode generators $L_{0}=L\left[\xi_{0}\right]$ and $\bar{L}_{0}=\bar{L}\left[\bar{\xi}_{0}\right]$ are "conformal charges" roughly analogous to energies.

Now consider any two-dimensional conformal field theory for which the lowest eigenvalues $\Delta_{0}$ and $\bar{\Delta}_{0}$ of $L_{0}$ and $\bar{L}_{0}$ are nonnegative. Cardy's striking result is that the asymptotic density of states at large conformal charge $(\Delta, \bar{\Delta})$ takes the simple form

$$
\ln \rho(\Delta, \bar{\Delta}) \sim 2 \pi \sqrt{\frac{\left(c-24 \Delta_{0}\right) \Delta}{6}}+2 \pi \sqrt{\frac{\left(\bar{c}-24 \bar{\Delta}_{0}\right) \bar{\Delta}}{6}},
$$

independent of any other details of the theory. This is precisely the kind of universal behavior we need.

\section{How to ask the right question}

Before proceeding further, we must confront a fundamental problem: how do we formulate our questions to ensure that we are asking about a black hole? In semiclassical computations, this is not an issue-we simply choose a fixed black hole geometry and analyze fields and metric fluctuations in that background. In a full quantum theory of gravity, though, we cannot do this- the components of the metric do not commute, and cannot be specified simultaneously. We must instead find new conditions strong enough to ensure the presence of a black hole, but weak enough to be allowed by quantum mechanics.

The simplest conditions of this sort are restrictions on the asymptotic behavior of the metric. The basic symmetry of general relativity is diffeomorphism invariance, manifested in the Hamiltonian formalism through a set of constraints - the "diffeomorphism constraints" $\mathcal{H}_{i}$ and the "Hamiltonian constraint" $\mathcal{H}_{\perp}$ - that generate coordinate transformations. When boundary conditions are imposed, these constraints acquire boundary terms, which can change their Poisson algebra. For the $(2+1)$ dimensional black hole, these terms lead to Virasoro algebra at infinity [19] that gives, via the Cardy formula, just the right enumeration of states to explain the BekensteinHawking entropy [20]. In general, though, asymptotic conditions are too weak-they cannot distinguish between a black hole and a "star" — and the results depend on particular features of $(2+1)$-dimensional spacetime that are not easily generalized. Many near-extremal black holes studied in string theory have near-horizon geometries that look $2+1$ dimensional, allowing one to apply this method [2], but clearly a more general approach would be desirable. 
One such generalization is to treat the horizon as a boundary-or, more precisely, as a hypersurface upon which we impose "boundary conditions". Once again, such restrictions alter the symmetry algebra of general relativity. Now, in any spacetime dimension greater than two, the result is a Virasoro algebra with the right central charge and conformal charges to yield the Bekenstein-Hawking entropy [21-23]. But the diffeomorphisms whose algebra leads to this result are generated by vector fields that blow up at the horizon [24,25], and this divergence is poorly understood.

The newest approach [26,27] is to impose the existence of a horizon as a constraint on initial values of gravitational degrees of freedom. We begin again with standard general relativity, but now add a set of "horizon constraints" $K_{\alpha}=0$ on an initial hypersurface. These may ensure, for example, that a chosen hypersurface has vanishing expansion and a prescribed surface gravity. Such horizon constraints will typically fail to commute with the diffeomorphism and Hamiltonian constraints, but we can cure this in a manner first suggested by Bergmann and Komar [28]: we add "zero," in the form of multiples of the $K_{\alpha}$, to $\mathcal{H}_{i}$ and $\mathcal{H}_{\perp}$ to produce new generators that commute with the $K_{\alpha}$. This will change the Poisson algebra of $\mathcal{H}_{i}$ and $\mathcal{H}_{\perp}$, potentially giving rise to the desired central charge. In [27], this program was carried out for a very general version of two-dimensional dilaton gravity, using the "radial quantization" techniques popular in string theory. The result was again a Virasoro algebra that yielded the correct Bekenstein-Hawking entropy. For now, this "horizon constraint" approach seems the most general; it appears to incorporate both the "horizon as boundary" results and the method of asymptotic symmetries.

\section{What are we counting?}

The advantage of the Cardy formula is that while it lets us counts states, it does not require detailed knowledge of the states being counted. Nevertheless, these results suggest an interesting effective description of black hole entropy.

In conventional quantum gravity, physical states are required to be invariant under diffeomorphisms; in our context,

$$
L[\xi] \mid \text { phys }\rangle=\bar{L}[\bar{\xi}] \mid \text { phys }\rangle=0 .
$$

If the Virasoro algebra (2) has a central charge, though, such conditions are incompatible with the Poisson brackets. We know how to weaken (4): we can require, for example, that only positive frequency modes annihilate physical states [29]. But then new states that had formerly been excluded-for instance, the "descendant states" $L_{-n}|0\rangle$-become physical.

This phenomenon is strongly reminiscent of the Goldstone mechanism [30]. The conformal anomaly breaks diffeomorphism invariance, and as a consequence, "wouldbe pure gauge" degrees of freedom become physical. For asymptotically anti-de Sitter spacetimes in three [31] and five [32] dimensions, an explicit description of the resulting degrees of freedom at infinity is possible; one might hope for something similar at a horizon. 
Perhaps the most important open question is whether Hawking radiation and black hole evaporation can also fit into this approach. As noted above, one can compute Hawking radiation with techniques that rely on conformal anomalies of the radiating matter fields $[15,16]$, and Emparan and Sachs have shown that in $2+1$ dimensions, a scalar field can be coupled to the conformal boundary degrees of freedom to obtain Hawking radiation [33]. If a similar mechanism could be found at the horizon, it would represent major progress.

Acknowledgments This work was supported in part by the Department of Energy grant DE-FG0291ER40674.

\section{References}

1. Strominger, A., Vafa, C.: Phys. Lett. B 379, 99 (1996) hep-th/9601029

2. Skenderis, K.: Lect. Notes Phys. 541325 (2000) hep-th/9901050

3. Mathur, S.D.: Fortsch. Phys. 53, 793 (2005) hep-th/0502050

4. Ashtekar, A., Baez, J., Corichi, A., Krasnov, K.: Phys. Rev. Lett. 80, 904 (1998) gr-qc/9710007

5. Livine, E.R., Terno, D.R.: Nucl. Phys. B 741, 131 (2006) gr-qc/0508085

6. Frolov, V.P., Fursaev, D.V.: Phys. Rev. D 56, 2212 (1997) hep-th/9703178

7. Rideout, D., Zohren, S.: Class Quant. Grav. 23, 6195 (2006) gr-qc/0606065

8. Hawking, S.W., Hunter, C.J.: Phys. Rev. D 59, 044025 (1999) hep-th/9808085

9. Bombelli, L., Koul, R.K., Lee, J., Sorkin, R.D.: Phys. Rev. D 34, 373 (1986)

10. Emparan, R.: JHEP 0606, 012 (2006) hep-th/0603081

11. Hawking, S.W.: Nature 248, 30 (1974)

12. Birmingham, D., Gupta, K.S., Sen, S.: Phys. Lett. B 505, 191 (2001) hep-th/0102051

13. Medved, A.J.M., Martin, D., Visser, M.: Phys. Rev. D 70, 024009 (2004) gr-qc/0403026

14. Jacobson, T., Kang, G.: Class. Quant. Grav. 10, L201 (1993) gr-qc/9307002

15. Robinson, S.P., Wilczek, F.: Phys. Rev. Lett. 95, 011303 (2005) gr-qc/0502074

16. Iso, S., Morita, T., Umetsu, H.: hep-th/0701272

17. Cardy, J.A.: Nucl. Phys. B 270, 186 (1986)

18. Blöte, H.W.J., Cardy, J.A., Nightingale, M.P.: Phys. Rev. Lett. 56, 742 (1986)

19. Brown, J.D., Henneaux, M.: Commun. Math. Phys. 104, 207 (1986)

20. Carlip, S.: Class. Quant. Grav. 22, R85 (2005) gr-qc/0503022 (review)

21. Carlip, S.: Phys. Rev. Lett. 82, 2828 (1999) hep-th/9812013

22. Carlip, S.: Class. Quant. Grav. 16, 3327 (1999) gr-qc/9906126

23. Cvitan, M., Pallua, S., Prester, P.: Phys. Rev. D 70, 084043 (2004) hep-th/0406186

24. Dreyer, O., Ghosh, A., Wisniewski, J.: Class. Quant. Grav. 18, 1929 (2001) hep-th/0101117

25. Koga, J.: Phys. Rev. D 64, 124012 (2001) gr-qc/0107096

26. Carlip, S.: Class. Quant. Grav. 22, 1303 (2005) hep-th/0408123

27. Carlip, S.: gr-qc/0702107

28. Bergmann, P.G., Komar, A.B.: Phys. Rev. Lett. 4, 432 (1960)

29. Di Francesco, P., Mathieu, P., Sénéchal, D.: Conformal Field Theory. Springer, Heidelberg (1997)

30. Kaloper, N., Terning, J.: personal communication

31. Carlip, S.: Class. Quant. Grav. 22, 3055 (2005) gr-qc/0501033

32. Aros, R., Romo, M., Zamorano, N.: hep-th/0612028

33. Emparan, R., Sachs, I.: Phys. Rev. Lett. 81, 2408 (1998) hep-th/9806122 